\title{
Controlling the process of muon formation for muon-catalyzed fusion: method of non-destructive average muon sign detection
}

\author{
Leif Holmlid* ${ }^{*}$
}

*Correspondence:

holmlid@chem.gu.se Atmospheric Science, Department of Chemistry and Molecular Biology,

University of Gothenburg, SE-412 96 Göteborg, Sweden

\begin{abstract}
The recent development of intense muon sources (Holmlid, Swedish Patent SE 539,684 C 2 (2017)) is crucial for the use of muon-catalyzed fusion reactors (L. Holmlid, Fusion Science and Technology 75, 208 (2019)) which are likely to be the first generation of practical fusion reactors. For this purpose, only negative muons are useful. For existing sources where negative muons can be ejected (if not formed) preferentially, it is necessary to know the amount of negative muons to determine and optimize the fusion reactor efficiency on-line. Here, a method is developed to measure the absolute muon flux and its average sign without collecting or deflecting the muons. The muons from the patented muon generator have an energy of $100 \mathrm{MeV}$ and above and an intensity of $10^{13}$ muons per laser pulse. Here, the detection of the relativistic laser-induced muons from $\mathrm{H}(0)$ is reported with a standard particle beam method, using a wire coil on a ferrite toroid as detector for the relativistic particles. The coil detection method shows that these relativistic particles are charged, thus not photons, neutrinos or neutral kaons. This makes the coil method superior to scintillator methods and it is the only possible method due to the large muon intensity. If an equal number of positive and negative mouns passed the coil, no signal would be observed. The signal at the coil in the case shown here is due to relativistic positive muons as concluded from a signal charge sign verification in the coil.
\end{abstract}

Keywords: Ultra-dense hydrogen, Ferrite toroid, Current coil

PACS: 67.63.Gh, 29.25.-t, 29.40.-n, 84.32.Hh

\section{Introduction}

Muons are observed from pulsed laser impact on ultra-dense hydrogen $H(0)$ [1-3] by plastic scintillators and solid converters, with the resulting electrons and positrons observed by photo-multipliers and MCA (multichannel analysis) energy spectral systems. $\mathrm{H}(0)$ (described in a review of 50 publications in ref. [4]) is here implied to mean both ultradense protium $\mathrm{p}(0)$ [5] and ultradense deuterium $\mathrm{D}(0)$ [4] and mixed forms $\mathrm{pD}(0)$. The (0) indicates the orbital angular momentum of the electrons in the material [4]. Linear Kurie plots show that the electrons observed are due to processes similar to beta decay or more likely due to lepton pair production $[6,7]$. These studies have led

(c) The Author(s), 2021 licensee Springer on behalf of EPJ. Open Access This article is licensed under a Creative Commons Attribution 4.0 International License, which permits use, sharing, adaptation, distribution and reproduction in any medium or format, as long as you give appropriate credit to the original author(s) and the source, provide a link to the Creative Commons licence, and indicate if changes were made. The images or other third party material in this article are included in the article's Creative Commons licence, unless indicated otherwise in a credit line to the material. If material is not included in the article's Creative Commons licence and your intended use is not permitted by statutory regulation or exceeds the permitted use, you will need to obtain permission directly from the copyright holder. To view a copy of this licence, visit http://creativecommons.org/licenses/by/4.0/. 
to the realization of a feasible fusion reactor using muon-catalyzed fusion [8]. A muon source for this type of reactor is patented [9]. Most muon generating processes including the patented source give both positive and negative muons, while only negative muons are useful for muon-catalyzed fusion. Muon sources using high-energy particle impact give muons through pair production, thus with equal numbers of positive and negative muons. The patented source [9] can give both negative and positive muons under various conditions, and is thus a better starting point for developing muon sources with mainly negative muons needed for highly efficient muon-catalyzed reactors.

For the development of even more efficient muon-catalyzed fusion reactors, it is necessary to measure the absolute flux of the muons and their sign of charge in working fusion reactors, without destruction of the muons and to do this at the actual high muon intensities of $>10^{13}$ per laser pulse [8]. The present contribution describes a coil method which can be used for this purpose, and gives the scientific background for its use.

The current due to muons is, besides the coil, also observed by metal collectors giving time-of-flight distributions with apparent particle energy of $10-500 \mathrm{MeV} \mathrm{u}^{-1}[10-$ 12]. This muon signal decays after the laser pulse with a time constant corresponding to the life-time of the precursor mesons $[13,14]$. Mesons and muons are detected as a result of the pulsed-laser impact. Mesons are detected in the same vacuum chamber [12] and muons are detected also outside the chamber at a few $m$ distance [2]. Spontaneous ejection of muons is also observed from $\mathrm{H}(0)$ [15]. Ultra-dense hydrogen $\mathrm{H}(0)$ is well described as the third (shortest distance) type of matter $[5,16]$ based on the quantum mechanical radius of the electron $r_{\mathrm{q}}=0.192 \mathrm{pm}$. The theoretical and experimental information describing $\mathrm{H}(0)$ is given in a recent review [4]. $\mathrm{H}(0)$ may be related to other Compton-scale materials that have been proposed to exist [17], but is otherwise a unique material, probably the most common material in the universe [18].

We have now applied a superior method to observe the relativistic charged particles ejected from $\mathrm{H}(0)$ at high intensity, namely an induction coil (current transformer) to directly observe the current of charged particles thus excluding all neutral particles like photons, neutrons, neutrinos and neutral kaons $K_{L}^{0}$ or $K_{S}^{0}$ as the relativistic signal. If positive and negative particles were emitted with equal probability, the signal in the coil would be zero. The coil method has been used previously for observing particle bunches in accelerators [19-21]. This method is here calibrated by direct current collection. This method also proves conclusively through current sign verification that the particle current is positive. No other method can do this with simultaneous identification of the precursor mesons at such high signal intensities. The nuclear processes giving the mesons and muons are already discussed elsewhere [3, 11-14].

Muon beams and muon generation are of interest at present for different experiments, mainly in large experiments like muon storage rings, muon colliders and muon cooling experiments (for example MICE) [22]. In muon spin spectroscopy ( $\mu \mathrm{SR}$, muon spin rotation), low-energy positive muons formed by decay of positive pions are implanted into materials [23]. For muon catalyzed fusion [24, 25], negative muons are needed [1, $2,12]$. A muon generator for this process is patented [9] and has been analyzed in detail [8]. The muon catalyzed fusion using this muon generator has been verified by neutron detection [26]. The properties of $\mathrm{H}(0)$ are of great importance for astrophysics and astronautics $[18,27,28]$. 


\section{Background}

The extensive information in the review paper [4] and in the 30 experimental studies on $\mathrm{H}(0)$ cannot be repeated here. Instead, the necessary background from these studies is summarized below. The properties of the nuclear particles involved are described in refs. $[6,7,29,30]$.

Ultra-dense hydrogen $\mathrm{H}(0)$ is characterized by electron orbital angular momentum $l=0$.

It consists mainly of chain clusters (molecules) of the form $\mathrm{H}_{2 N}(0)$. Several different spin states exist with $s=2$ most commonly observed [4]. This $\mathrm{H}(0)$ state which is deposited on the laser target [31] in the present experiments has an $\mathrm{H}-\mathrm{H}$ bond distance of $2.3 \mathrm{pm}$ at $s=2$. The laser pulse interacts with the loosely bound electrons in the two forms of hydrogen matter which have the largest typical dimensions [5, $16]$ and which are easily formed from $\mathrm{H}(0)$. This laser interaction brings small nonsuperfluid clusters $\mathrm{H}_{3}(0)$ and $\mathrm{H}_{4}(0)$ [32] into the $s=1$ state at $0.56 \mathrm{pm}$ interatomic distance [4]. At this distance, the nuclear processes are likely to take place within $1 \mathrm{~ns}$, as in muon catalyzed fusion with a similar internuclear distance [25]. These nuclear processes [3, 11-14] form charged and neutral kaon pairs and charged pion pairs [33]. The short-lived neutral kaons decay within 1 ns mainly to pion pairs with high kinetic energy. The other kaon types decay much slower at 12.4 ns (charged) and 52 ns (longlived neutral) decay time to various pions and muons. The muons have long decay time, at $2.20 \mu \mathrm{s}$ at rest and relativistic velocity which means that they are the ones that move to the detection systems (coil, collectors) at 1-2 m distance (see further below).

\section{Muon signal}

Unfortunately, no selective method exists for detecting muons and none has been developed despite that muons have been known since 1936.

The requirements on the method for muon detection needed for the use as muon monitor in fusion reactors are several and quite difficult to satisfy simultaneously:

1) a largely linear response beyond $10^{9}$ muons per laser pulse of $5 \mathrm{~ns}$ width thus beyond $2 \times 10^{8}$ muons per ns,

2) a response to the sign of the muons since only the negative muons can give fusion,

3) a response largely independent of the kinetic energy of the muons. Different meson decay channels give muons with different energy, and all muons need to be thermalized before giving fusion in the reactor, so it is only the number of muons which is of interest,

4) the method should be non-destructive so the muon flux can be monitored without stopping or decreasing the rate of fusion.

All these requirements exclude any method based on ionization or excitation like scintillator methods. Only direct particle methods like measuring the current of the particles by a coil [19-21] as used here can cope with these requirements. The use of a coil in the beam is a standard method for measuring particle beams [21].

To prove that the experiments here demonstrate the behaviour of a bunch of muons, as is necessary for the evaluation of the coil function, requires slightly different 
methods than for the flux monitoring. These methods will be summarized below. Of course, not all of these methods have been used simultaneously but they have all been used repeatedly in published studies with laser interaction at the $\mathrm{H}(0)$ source.

The most common method for fast particle detection employs plastic scintillators with detection of the photons from the particle-scintillator interaction in a photo-multiplier tube (PMT) or similar device. This method has the large problem that it is not selective for muons, and other fast charged particles like mesons (pions and kaons) and leptons (electrons and positrons) can give similar signals. Another important problem can be the timing with PMT delay and rise times which are often of the order of a few ns. This is longer than for direct current detection via metallic collectors or coils. The sign of the muon cannot be derived from such detection, and just a few muons can be detected within the time span of 5 ns due to pulse overlap and signal saturation. This is many orders of magnitude lower than the signal levels observed from the muon generators using $\mathrm{H}(0)$ [8].

The only published effort [2] to develop a selective detection method for muons is from our group, using metallic converters to give lepton pair production and using the MCA energy spectra for characterizing the lepton energy distributions. A few publications have used this method successfully $[1,2,15]$. It has thus been proved that muons are formed in the system studied here, from the laser interaction with ultradense hydrogen $\mathrm{H}(0)$.

A few other methods exist to identify a signal as due to muons. The most direct is to observe the decay of the muons and measure their decay life-time. This has been done in the system of interest here, with laser-induced nuclear processes in $\mathrm{H}(0)$. The decay lifetime of the muons was directly measured by a pulse-counting PMT and MCS (multi-channel scaler) analysis, which gave good agreement with the best values for muons [3]. Thus, muons are formed in these experiments using $\mathrm{H}(0)$. They are formed by decay of several different mesons, notably charged and neutral kaons and charged pions $[6,7,29,30]$.

In the present experiment, the process giving the muons is further identified from the decay lifetime of their precursors, in this case from charged kaons with their typical decay time of $12.4 \mathrm{~ns}[6,7,29,30]$. This method has been used previously [12-14].The signal observed at a collector or a coil is due to a final particle $\mathrm{N}$ from the decay of an intermediate particle $\mathrm{M}$ which is formed and decays like $\mathrm{A} \rightarrow \mathrm{M} \rightarrow \mathrm{N}$ with this process triggered by the laser pulse on $\mathrm{H}(0)$. The time dependence of the signal of $\mathrm{N}$ is easily derived from the rate equations for $\mathrm{A} \stackrel{k_{1}}{\longrightarrow} \mathrm{M} \stackrel{k_{2}}{\longrightarrow} \mathrm{N}$

$$
\begin{aligned}
& -\frac{d n_{\mathrm{A}}}{d t}=k_{1} n_{\mathrm{A}} \\
& \frac{d n_{\mathrm{M}}}{d t}=k_{1} n_{\mathrm{A}}-k_{2} n_{\mathrm{M}} \\
& \frac{d n_{\mathrm{N}}}{d t}=k_{2} n_{\mathrm{M}}
\end{aligned}
$$

as

$$
n_{N}=\frac{k_{1} k_{2}}{k_{2}-k_{1}} n_{\mathrm{A} 0}\left(e^{-k_{1} t}-e^{-k_{2} t}\right)
$$


where $n_{\mathrm{A} 0}$ is the number density of the precursor A at time $t=0$ thus during the laser pulse. Equation (4) assumes that the initial number densities of $\mathrm{M}$ and $\mathrm{N}$ are zero. Here A is located at the target, $\mathrm{M}$ is a meson outside the target here a charged kaon, and $\mathrm{N}$ is the fast more longlived particle which can reach the coil or collector, thus a muon.

The decay spectrum of charged kaons is well known $[6,7,29,30]: 64 \%$ of the charged kaons decay to muons, $27 \%$ of the charged kaons give charged pions instead of muons, and such pions decay with a lifetime of 26 ns to muons. Some neutral pions are also formed which give gamma photons and no muons.

One further conclusive method for muon detection has also been used, by detecting neutrons from muon catalyzed fusion in $\mathrm{D}_{2}$ gas [26].

A more general particle method using magnetic deflection coupled to time-of-flight has also been used to study the meson and muon bunches [12]. This study shows that the particles have masses below $1 \mathrm{u}$ (thus they are not protons) and much higher than electrons or positrons. No distinction could be made between light mesons and muons in this study since the flux at the deflecting magnets, close to the $\mathrm{H}(0)$ covered source, certainly could contain both charged kaons, charged pions and muons. That study further showed that many of the fast particles were neutral, thus presumably long-lived neutral kaons. This has been verified in further experiments with decay-time measurements [33].

All these methods give the evidence that muons are formed, clearly from decay of mesons which are emitted from the laser interaction with ultradense hydrogen $\mathrm{H}(0)$. The decays of the various mesons have also been studied. The measurements show the correct decay-times [33] for charged pions, charged kaons, and neutral long-lived kaons with a precision of better than $1 \%$. Neutral pions and short-lived neutral kaons have too short lifetimes to be measured with high timing precision even if they can be observed to exist by other methods (to be published).

\section{Experimental}

The apparatus used has been described in several publications, for example in Ref. [34]. It has base pressure below $1 \times 10^{-6}$ mbar with $1000 \mathrm{~s}^{-1}$ diffusion pumping speed and is shown schematically in Fig. 1. The detector part is pumped separately. The central source part is described in Ref. [31]. The $\mathrm{H}(0)$ emitter is a cylindrical (extruded) sample of a porous iron oxide catalyst doped with $\mathrm{K}[35,36]$, a so called styrene catalyst (obsolete type Shell S-105). A styrene catalyst is an efficient hydrogen abstraction and transfer catalyst used in a large scale in the chemical industry. The emitter is mounted in the tight-fitting opening of a Pt tube. This metal tube is heated to $<400 \mathrm{~K}$ by a low-voltage AC current through its wall from end to end. Deuterium gas (99.8\%) is admitted through the tube at a pressure up to $1 \times 10^{-5}$ mbar into the chamber. The laser target $\mathrm{Ni}$ foil is placed at $45^{\circ}$ to the vertical direction and approximately $1 \mathrm{~cm}$ below the source tip. It has short Pt and Ir rods with $2 \mathrm{~mm}$ diameter spot-welded on it, to increase the catalytic effect for hydrogen splitting and to prevent the laser from boring through the target foil. This target is initially at room temperature.

A Nd:YAG laser with an energy of $<125 \mathrm{~mJ}$ in $5 \mathrm{~ns}$ long pulses at $10 \mathrm{~Hz}$ repetition rate is used at $532 \mathrm{~nm}$ (Gaussian profile). The laser beam is focused on the target at the 


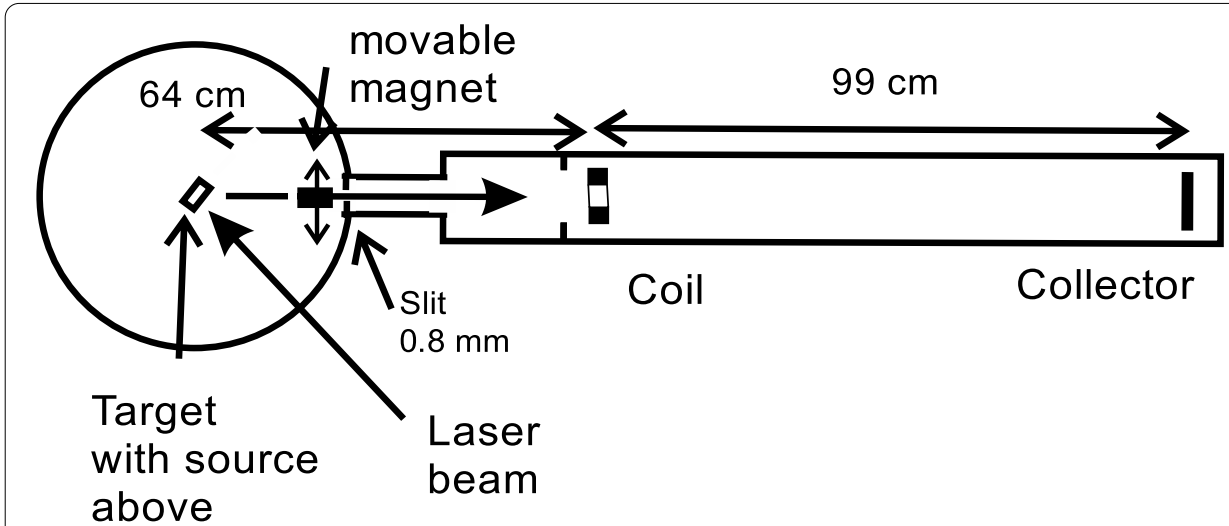

Fig. 1 Horizontal cut through the apparatus. The coil—collector part direction is at $45^{\circ}$ relative to the incoming laser beam

center of the chamber with an $f=400 \mathrm{~mm}$ spherical lens. This lens is mounted in a vertical motion translation stage in air. The intensity in the beam waist of (nominally) $70 \mu \mathrm{m}$ diameter is relatively low, $\leq 10^{12} \mathrm{~W} \mathrm{~cm}^{-2}$ as calculated for a Gaussian beam. The laser pulse is very clean with no disturbing intensity after the main pulse. This is certified by 10 years of studies using it for TOF-MS studies in easily photo-ionized alkali Rydberg Matter [37], where any such problems would have been clearly observed. The particle signal from the coil at $64 \mathrm{~cm}$ distance and from the collector at $163 \mathrm{~cm}$ distance from the target is alternately collected on a fast digital oscilloscope (Tektronix TDS 3032, $300 \mathrm{MHz}$, rise time $1.2 \mathrm{~ns}$ ) using the same cable. The outer collector consists of 2 layers of $20 \mu \mathrm{m} \mathrm{Al} \mathrm{foil} \mathrm{over} \mathrm{a} \mathrm{steel} \mathrm{ring} \mathrm{to} \mathrm{minimize} \mathrm{trapping} \mathrm{of} \mathrm{charged} \mathrm{particles} \mathrm{in} \mathrm{a} \mathrm{thick} \mathrm{col-}$ lector. This decreases the secondary electron emission and confusing particle decay in the collector. Its diameter is around $30 \mathrm{~mm}$. The collector is at a negative bias voltage of -24 or $-50 \mathrm{~V}$ by batteries in a shielded metal box (with coaxial connectors at both ends). In this way, secondary electrons from the collector are ejected promptly (they cannot return) and stray secondary electrons due to mesons, muons or gamma photons from other parts in the apparatus are rejected.

The measuring coil is wound on a N30 MnZn soft ferrite toroid with epoxy cover (EPCOS) with inner diameter $25 \mathrm{~mm}$. There are 19 turns of enameled copper wire wound on it in the negative direction. It hangs freely on the conductor wires in the particle beam. One end is connected to the $50 \Omega$ oscilloscope input, while the other end is connected to a $50 \Omega$ termination to the flange on the grounded chamber. See Fig. 2 . The coil used for detection of the relativistic particles from the laser-target interaction is a quite uncritical component [21]. This is the same construction which was used and analyzed in Ref, [38].

In the experiments, the signal rise time in the coil is observed to be $<1 \mathrm{~ns}$. This is seen for example from Fig. 4 where the rise times at both the collector and the coil are $4 \mathrm{~ns}$ within $1 \mathrm{~ns}$, thus with no longer risetime from the coil. The rise time at the collector is mainly due to the laser-pulse rise time of $3 \mathrm{~ns}$ and the rise time of the oscilloscope of $1.2 \mathrm{~ns}$, together giving a pulse rise time of $3.2 \mathrm{~ns}$ or less than $4 \mathrm{~ns}$ as seen in Fig. 4. The rise time of the coil should be equal to $\tau_{\text {rise }}=\sqrt{L_{s} C_{s}}$ as given in Ref. [21], with the stray capacitance $C_{s}$ and the stray inductance $L_{\mathrm{s}}$ due to the attached cable from the coil. 


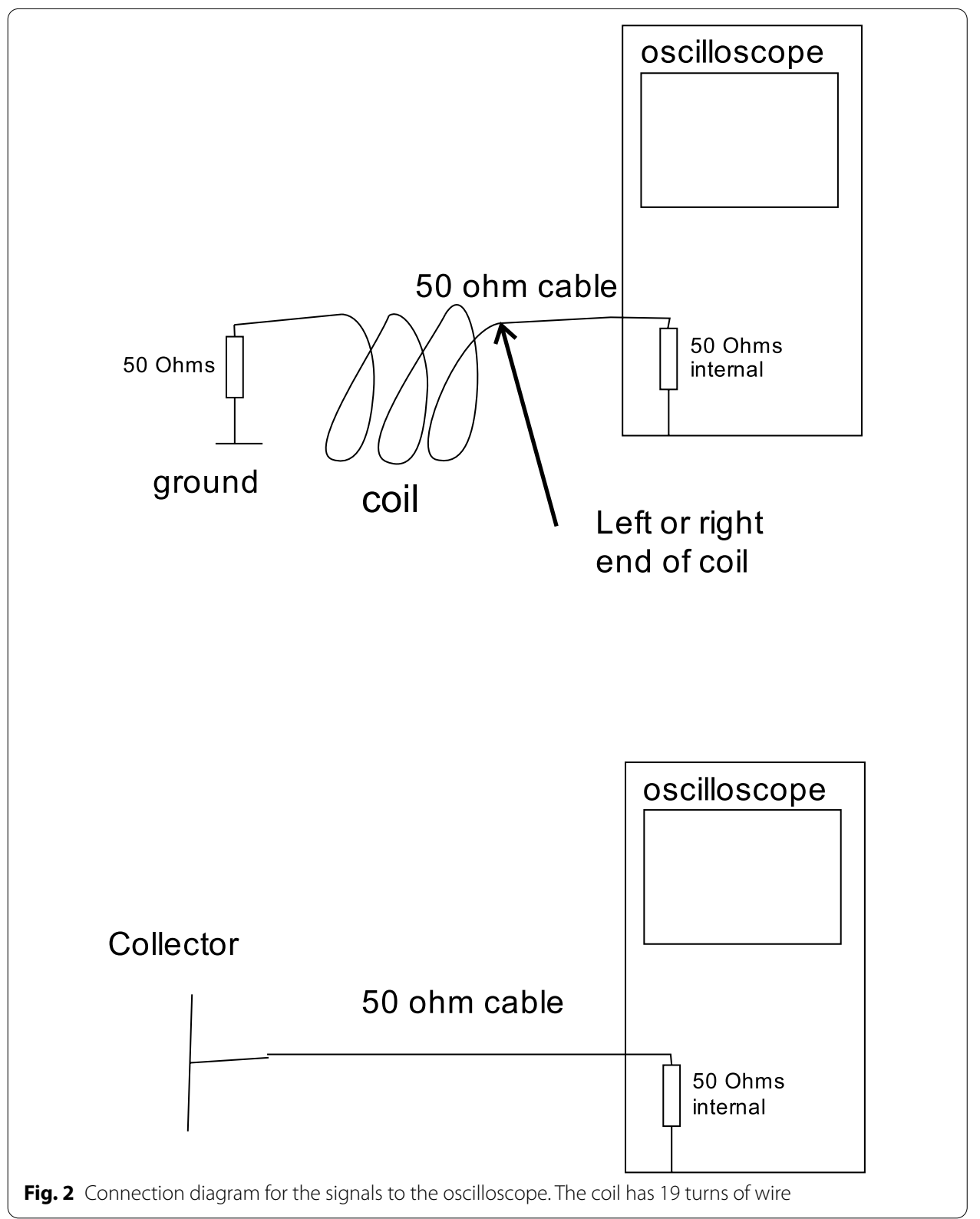

The RG58 cable used has approximately $0.24 \mu \mathrm{H} / \mathrm{m}$ and $100 \mathrm{pF} / \mathrm{m}$. This gives a value of $\left(0.24 \times 10^{-16}\right)^{0.5}=0.5 \times 10^{-8} \mathrm{~s}=5 \mathrm{~ns}$ with $1 \mathrm{~m}$ cable attached. However, this formula does not take into account the transmission cable with termination which will decrease the rise time strongly. So the observed rise time of $1 \mathrm{~ns}$ or less is valid. The other typical time for the coil, the droop time is equal to $\tau_{\text {droop }}=L / R_{L}$ [21], With a coil inductance $L$ of the order of $100 \mu \mathrm{H}$ and the load resistance $R_{L}$ at $100 \Omega$, the droop time becomes $1 \mu \mathrm{s}$ thus safely much longer than the decay times of interest here. The measurements here are differential and eliminate common mode noise as described in Ref. [21]. The signal subtraction procedure is clearly demonstrated in Fig. 3.

A pair of magnets (ferrite Y28 magnets with length $30 \mathrm{~mm}$, width $10 \mathrm{~mm}$ and height $5 \mathrm{~mm}$, remanence $400 \mathrm{mT}$ ) are mounted on an arm in the main chamber. See Fig. 1. 


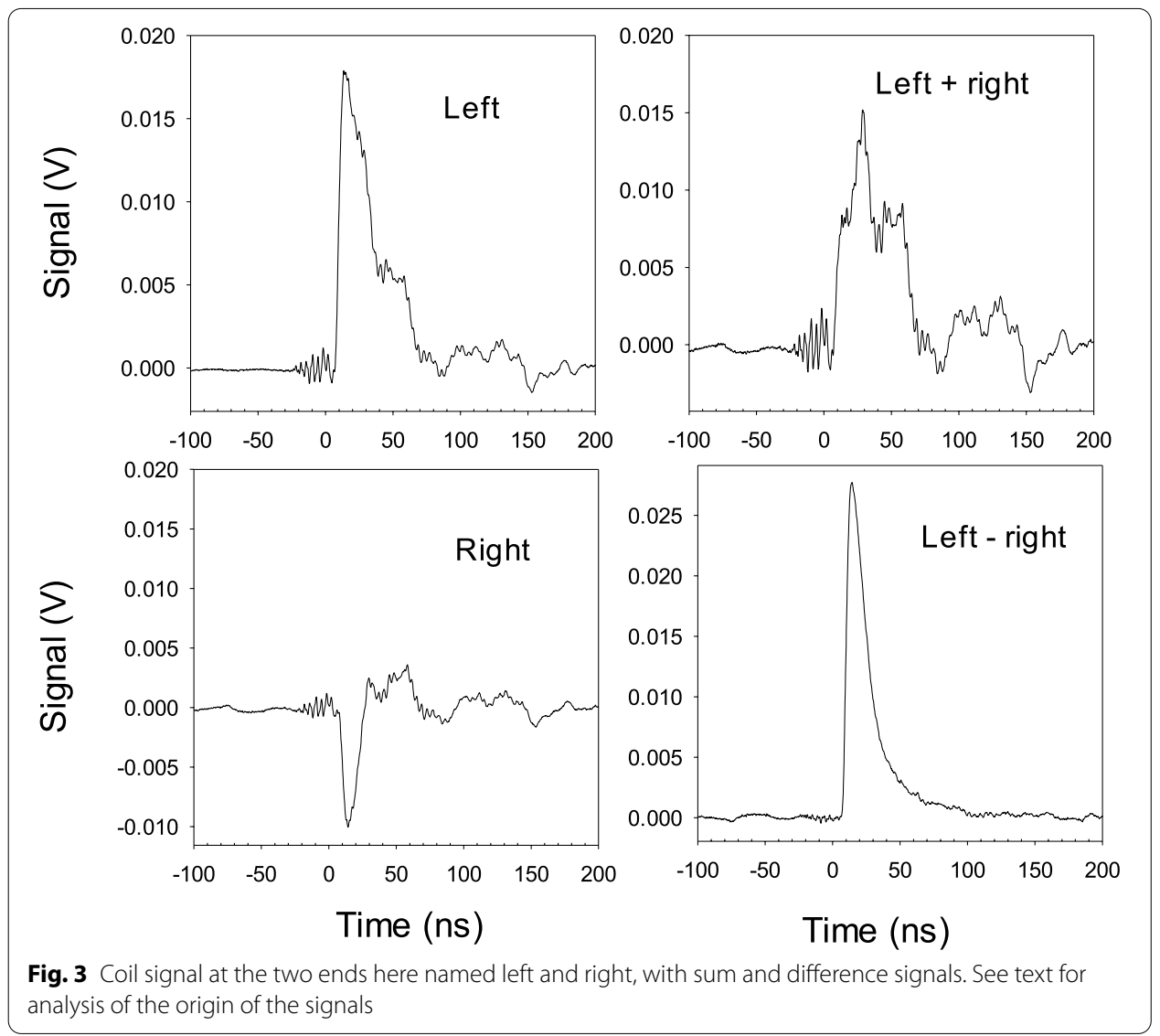

This pair of magnets can be moved in and out from the particle beam which passes to the detector part through the slit with a width of $0.8 \mathrm{~mm}$. The field strength between the magnets where the beam passes was $100 \mathrm{mT}$. The magnets are used to deflect any electrons and positrons out from the beam which moves through the narrow slit to the detector chamber. The deflection for electrons with energy $5 \mathrm{MeV}\left(10 \mathrm{GeVu}^{-1}\right)$ and thus velocity $0.99 \mathrm{c}$ in this magnetic field is calculated to be $2 \mathrm{~cm}$ at the collector position, thus deflecting outside the collector area. Almost the same setup was used in Ref. [12], and deflections for relativistic mesons and muons were given in Figs. 8, 19 and 25 in that reference. A muon in the present experiment with energy $1 \mathrm{GeV}\left(10 \mathrm{GeVu}^{-1}\right)$ is only deflected $0.1 \mathrm{~mm}$ in comparison.

\section{Results and discussion}

The experiments are started by optimizing the intensity of the signal to the outer collector by changing the laser position on the target and by changing the target position along the laser beam. See Fig. 1. The signal to the collector and to the two ends of the coil are then measured. See Fig. 2. The magnet holder is flipped into the beam to ensure that the beam does not contain electrons or positrons. Since a signal from impinging penetrating particles like gamma photons into the coil and possibly also from an electric field from the plasma could possibly be observed at the coil, the difference between the signals from the two ends of the coil is determined. This means 
that with a true particle-induced current signal in the coil into the oscilloscope 50 $\Omega$ input as $V_{\mathrm{c}}$ and a signal to the coil by impinging particles and an electric field for example due to the plasma as $V_{\mathrm{d}}$, the left-hand end of the coil gives $V_{\mathrm{L}}=V_{\mathrm{c}}+V_{\mathrm{d}}$, while the right-hand end of the coil gives $V_{\mathrm{H}}=-V_{\mathrm{c}}+V_{\mathrm{d}}$. Thus, one finds the difference $V_{\mathrm{L}}-$ $V_{\mathrm{H}}=2 V_{\text {c. }}$. The left-hand end giving a positive signal with a positive current through the coil is the "positive" signal from which the signal at the other end is subtracted. The sum of the two signals is $V_{\mathrm{L}}+V_{\mathrm{H}}=2 V_{\mathrm{d}}$. Due to the total load of $25 \Omega$ the factor of 2 is directly compensated for. See Fig. 2 . The sign of the current through the coil is found from separate experiments in air employing a square-wave current with a few ns rise time in a single conductor through the coil. The dependence of the winding direction of the wire coil on the sign of the signal is thus determined experimentally.

In Fig. 3, the signals at the two ends of the coil are shown and their difference and sum are found. The sum of the two signals has its first peak at longer time than the difference signal. The sum is apparently mainly due to the plasma at the target. The difference signal is always a smooth curve with a close to exponential decay, from now on called the coil signal. This signal shows that a true particle current passes through the coil. This signal disappears immediately when the valve to the coil-collector part is closed or if the magnets in the holder block the beam, as does also the collector signal. This proves directly that the signal is not caused by any electronic disturbance. The charge of the particles is positive as described further below. Photons will not give any signal current in the coil. If gamma photons create some kind of signal by penetrating into the coil and ejecting electrons there, this will be part of the signal $V_{\mathrm{d}}$ as described above, and their contribution will be removed by the difference procedure described. That the signals disappear when the valve to the detector part is closed does not mean that the muons in the beam are stopped by the steel plate in the valve but more likely that they are scattered out from the central beam by collisions in the steel plate. A small loss in energy by these collisions will also delay the muons to longer times on the oscilloscope screen so they will appear to be lost from the signal. Another contributing factor to the signal disappearance may also be that the beam signal at the valve is still mainly in the form of charged pions and kaons, which will interact more strongly with the steel plate than what the muons do. Assuming a velocity of $0.1 \mathrm{c}$ of these slower particles means a few ns to reach the coil, thus much shorter time than their decay time of $>12 \mathrm{~ns}$. The stronger interaction with a valve or beam flag of the mesons relative to the muons they produce has been observed in other experiments previously [3].

In Fig. 4, the signals measured simultaneously at the coil and the collector are compared. The time difference of the signal rises at the coil and the collector is only $2 \mathrm{~ns}$, and it is removed in the lower plot by shifting the time zero by $2 \mathrm{~ns}$. This brings the two signal curves into quite good agreement, with some slower signal observed at the collector. Thus, the particles are relativistic, moving $1 \mathrm{~m}$ distance in a few ns, thus with velocity close to $c$. Photons do not give any signal in the coil. With a metal collector instead of the coil at the position of the coil, the difference in time between the two collector signals is always $3-4 \mathrm{~ns}$. The smaller $2 \mathrm{~ns}$ shift observed here indicates that the coil signal is delayed 1-2 ns relative to the collector signal. This is found repeatedly also in another experimental set-up with the same type of coil detector. 


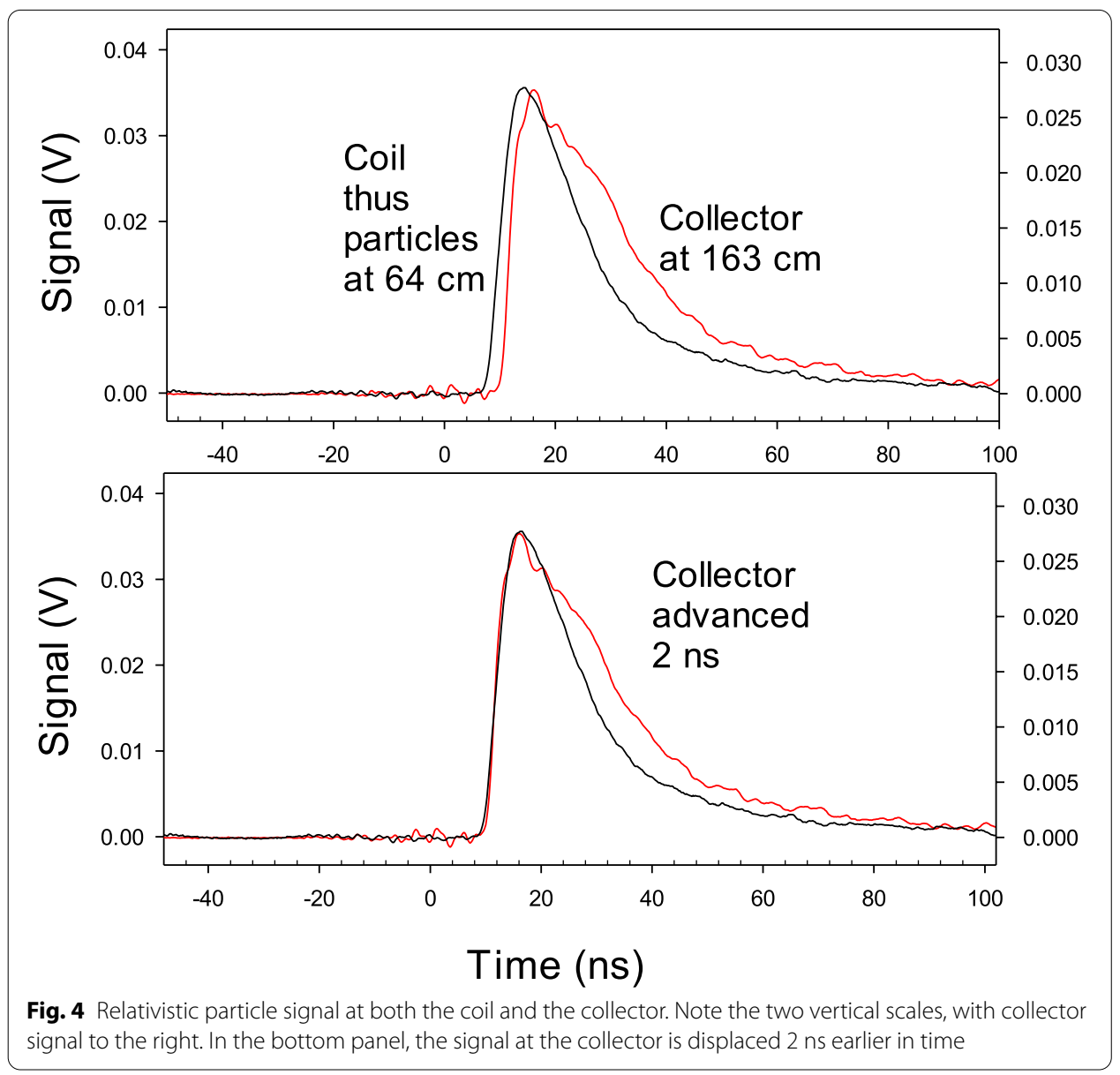

In Fig. 5, the coil and collector signals are interpreted as due to an intermediate meson $\mathrm{M}$, here a kaon or pion in a decay chain $\mathrm{A} \rightarrow \mathrm{M} \rightarrow \mathrm{N}[13,14,39]$. A more detailed derivation of the signal time variation is given in the Muon signal section. A is a precursor entity for example a small $\mathrm{H}(0)$ cluster. $\mathrm{N}$ is thus the almost stable long-lived type of particle (in this case a muon) giving the observed signals at the coil and collectors. The rate constants are $k_{1}$ and $k_{2}$ for the two reaction steps. The number density of $\mathrm{M}$ mesons is given by $[13,14]$

$$
n_{\mathrm{M}}=\frac{k_{1}}{k_{2}-k_{1}} n_{\mathrm{A} 0}\left(e^{-k_{1} t}-e^{-k_{2} t}\right)
$$

where $n_{\mathrm{A} 0}$ is the number density of the precursor A at time $t=0$ thus during the laser pulse. The corresponding decay time constants $\tau=1 / k$ are used below. It is observed that the decay time constant $\tau_{2}$ of $12 \mathrm{~ns}$ in Fig. 5 agrees with that for charged kaons, as found previously using two collectors in line [13]. In many experiments also a contribution from charged pion decay is observed. This is also observed in other experiments of the present type. The longer decay observed in the tail of the collector signal in Fig. 5 


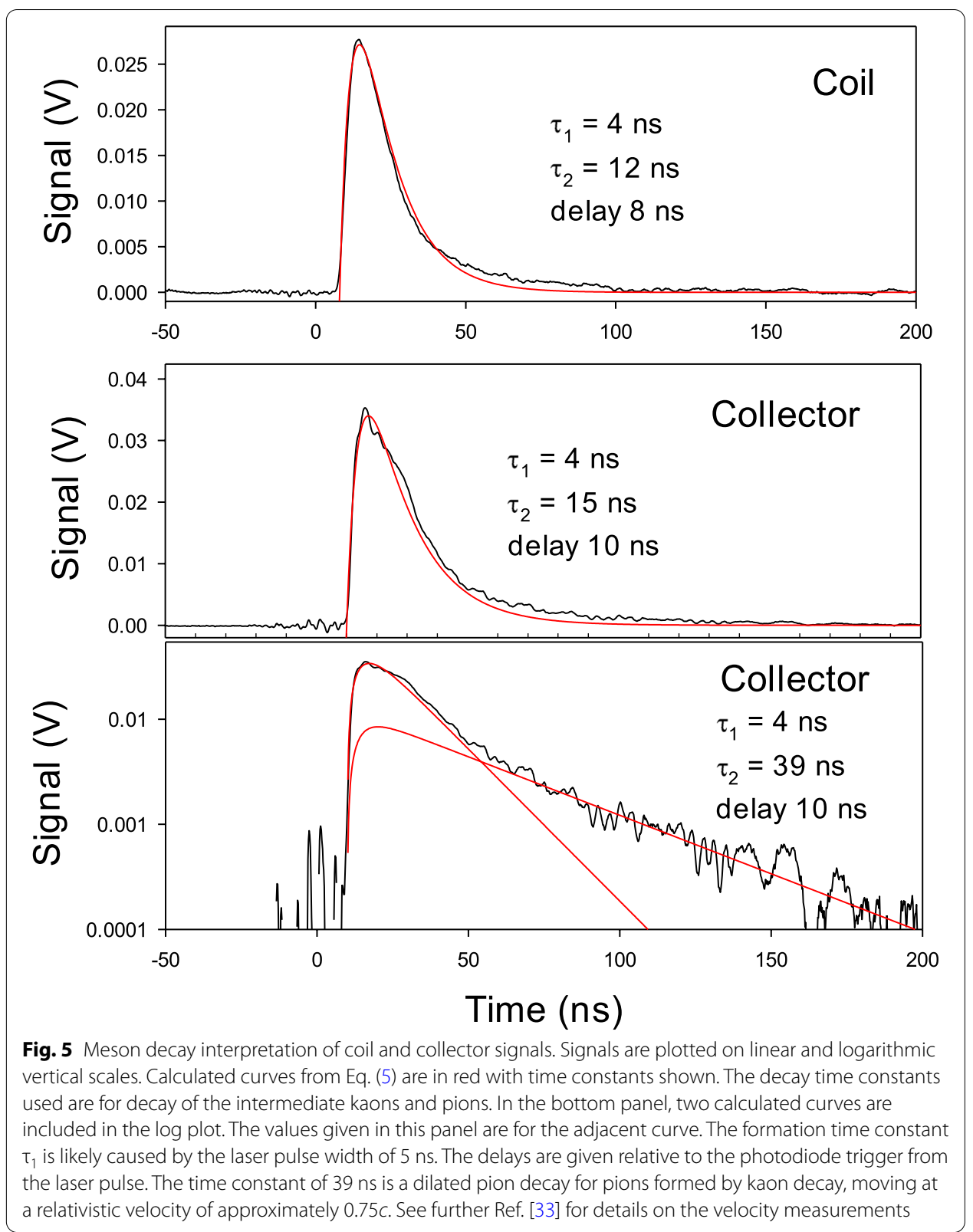

corresponds to relativistic dilation of the charged pion decay time of $26 \mathrm{~ns}$. The velocity of these pions is close to $0.75 c$ [33].

The present experiments show relativistic positive muons in the beam flux from the source. High precision decay time constants for the mesons have been published previously, see refs. [12, 33, 38].

The signal at the collector directly gives the peak of the signal distribution, of the order of $1 \mathrm{~mA}$ from the input resistance of $50 \Omega$. Thus this current gives a calibration of the peak signal in the coil at $0.03-0.05 \mathrm{~V} \mathrm{~mA}^{-1}$. This is similar to published results for coil detection of electron pulses in electron accelerators $[19,20]$. This shows that the pulse transformer i.e. the coil is working correctly. Since the signals at the collector and the 
coil are similar in width as shown in Fig. 4, no large extra signal contribution exists at the collector.

The derivation of the signal time variation in the Muon signal section describes the observed signal well. The first point to understand from the results is of course that the signal decaying with a certain time constant is not the signal created at the collector or coil by the decaying type of particle, but created at the coil or collector by a particle formed by the decaying particle. Any other interpretation is of course impossible. After kaons and pions decay to muons, the longer-living muons give the signal observed at the coil and collector and this signal decreases in time since the number of decaying pions at the target decreases with their characteristic decay time.

The decaying current observed in the same way by the coil and the collector is due to decaying particles which are formed with relatively low velocity in the target region. If the particles detected were decaying in the beam, they could not be observed as decaying at both the coil and at the collector. Thus, the particles moving in the beam are relatively long-lived and are formed by decay from other particles with decay times of 12 and $26 \mathrm{~ns}$ as observed in the matching of the results in Fig. 5 and in previous studies [12-14]. Muons from pions and kaons are thus the final particles observed by the coil and the collectors. The direct measurement by the coil shows that the current here is positive. In similar coil experiments at higher hydrogen pressure with $\mathrm{p}(0)$, the signal is normally negative and concluded to be due to negative muons. This agrees with the results on muon decay from $\mathrm{H}(0)$ [3].

The expected muon energies can be estimated $[7,30]$. The kaons formed initially at the target often have relatively low kinetic energy [3, 11-14]. The main decay channel for the charged kaons, exemplified as $\mathrm{K}^{+} \rightarrow \mu^{+}+$a muon neutrino, gives up to $388 \mathrm{MeV}$ to the muon. Such muons, which are related to the observed decay time of $12 \mathrm{~ns}$, thus have high energy and a velocity up to $0.98 c$. If the pions formed initially are relatively slow, the muons formed by pion decay have up to $34 \mathrm{MeV}$ kinetic energy, or a velocity of up to $0.65 c$. With fast initial precursor pions, the muon energy related to the $26 \mathrm{~ns}$ decay time will be even higher. Thus, relativistic muons are expected.

The particles in the beam formed by the decay of kaons and pions in the experiments are muons $[3,6,7,29,30]$. Of course, the muons further decay to electrons and positrons, but that happens mainly outside the apparatus used. The lifetime of relatively slow such particles from meson decay in the flux from $\mathrm{H}(0)$ has been measured in a previous publication [3] and agrees with the known muon lifetime. These positive muons had been slowed down by numerous collisions in solid matter. Here, the possibility that the direct relativistic signal from the meson decay is due to photons or other neutral particles like neutral kaons or neutrinos is disproved directly by the use of a coil to verify that the particles are charged and indeed positive in this case. The coil method used here is superior to other muon detection methods in several respects. That the sign of the particles can be observed is of course crucial for the use as muon monitor. It should be noted that no signal will be observed by the coil if equal numbers of positive and negative particles pass through it simultaneously.

Finally, it may be of interest to estimate the number of particles ejected from the target. The signal peak in Fig. 4 to the collector at $163 \mathrm{~cm}$ distance from the target contains of the order of $3 \times 10^{7}$ charges per laser shot. Assuming that each muon ejects just one 
electron from the collector and that each meson gives one muon, this gives a total number of ejected mesons at the target of the order of $10^{12}$ per laser shot, using the small opening angle of the collector.

This number should be increased due to the angular restrictions imposed by the $0.8 \mathrm{~mm}$ slit, but this cannot be done accurately since the laser position on the target relative to the slit is not known with a precision better than $0.1 \mathrm{~mm}$. The assumption that the ejection from the target is isotropic is based on the likely random directions of the decays of the mesons and the relativistic velocity of the muons. The value of $10^{12}$ muons formed per laser shot is slightly lower than previous estimates of $10^{13}$ [8] at optimum conditions, probably due to the partial interception by the narrow slit in the present experiments.

\section{Acknowledgements}

Part of the equipment was constructed and built with support from GU Ventures AB, The Holding Company at University of Gothenburg. Open access funding provided by University of Gothenburg.

\section{Author's contributions}

The author(s) read and approved the final manuscript.

\section{Funding}

Open access funding provided by University of Gothenburg.

\section{Availability of data and materials}

The data that supports the findings of this study are available within the article and can also be obtained from the author on reasonable request.

\section{Declarations}

Competing interests

The author declares that he has no competing interests.

Received: 15 April 2021 Accepted: 18 August 2021

Published online: 04 October 2021

\section{References}

1. Holmlid L, Olafsson S. Charged particle energy spectra from laser-induced processes: nuclear fusion in ultra-dense deuterium D(0). Int J Hydr Energy. 2016;41:1080.

2. Holmlid L, Olafsson S. Muon detection studied by pulse-height energy analysis: novel converter arrangements. Rev Sci Instrum. 2015;86:083306. https://doi.org/10.1016/j.heliyon.2019.e01864

3. Holmlid L, Olafsson S. Decay of muons generated by laser-induced processes in ultra-dense hydrogen. Heliyon. 2019;5(6):e01864. https://doi.org/10.1016/j.heliyon.2019.e01864.

4. Holmlid L, Zeiner-Gundersen S. Ultradense protium $\mathrm{p}(0)$ and deuterium $\mathrm{D}(0)$ and their relation to ordinary Rydberg matter: a review. Phys Scr. 2019;74(7). https://doi.org/10.1088/1402-4896/ab1276.

5. Holmlid L. Excitation levels in ultra-dense hydrogen $\mathrm{p}(-1)$ and $\mathrm{d}(-1)$ clusters: structure of spin-based Rydberg Matter. Int J Mass Spectrom. 2013;352:1. https://doi.org/10.1016/j.ijms.2013.08.003.

6. Krane KS. Introductory nuclear physics. Hoboken: Wiley; 1988.

7. Burcham WE, Jobes M. Nuclear and particle physics. Harlow: Pearson; 1995.

8. Holmlid L. Existing source for muon-catalyzed nuclear fusion can give MW thermal fusion generator. Fusion Sci Technol. 2019;75(3):208-17. https://doi.org/10.1080/15361055.2018.1546090.

9. Holmlid L. Apparatus for generating muons with intended use in a fusion reactor. Swedish Patent nr SE 539684 C 2. Published 2017-10-31.

10. Holmlid L. Direct observation of particles with energy $>10 \mathrm{MeV} / \mathrm{u}$ from laser-induced processes with energy gain in ultra-dense deuterium. Laser part Beams. 2013;31:715. https://doi.org/10.1017/S0263034613000414.

11. Holmlid L. Two-collector timing of 3-14 MeV/u particles from laser-induced processes in ultra-dense deuterium. Int J Modern Phys E. 2013;22:1350089. https://doi.org/10.1142/S0218301313500894.

12. Holmlid L. Mesons from laser-induced processes in ultra-dense hydrogen H(0). PLoS One. 2017;12:e0169895. https:// doi.org/10.1371/journal.pone.0169895. The originally published file can be found at https://gup.ub.gu.se/file/ 208005 and other locations on the web.

13. Holmlid L. Nuclear particle decay in a multi-MeV beam ejected by pulsed-laser impact on ultra-dense hydrogen H(0). Int J Modern Phys E. 2015;24:1550080. https://doi.org/10.1142/S0218301315500809.

14. Holmlid L. MeV particles in a decay chain process from laser-induced processes in ultra-dense deuterium $D(0)$. Int J Modern Phys E. 2015;24:1550026. https://doi.org/10.1142/S0218301315500263. 
15. Holmlid L, Olafsson S. Spontaneous ejection of high-energy particles from ultra-dense deuterium D(0). Int J Hydr Energy. 2015;40:10559. https://doi.org/10.1016/j.ijhydene.2015.06.116.

16. Hirsch JE. The origin of the Meissner effect in new and old superconductors. Phys Scr. 2012;85:035704. https://doi. org/10.1088/0031-8949/85/03/035704.

17. Mayer FJ, Reitz JR. Electromagnetic composites at the Compton scale. Int J Theor Phys. 2012;51:322. https://doi.org/ 10.1007/s10773-011-0959-8.

18. Holmlid L. Ultra-dense hydrogen $\mathrm{H}(0)$ as dark matter in the universe: new possibilities for the cosmological red-shift and the cosmic microwave background radiation. Astrophys Space Sci. 2019;364:141. https://doi.org/10.1007/ s10509-019-3632-y.

19. Millard JK, Lewis TA, Allin GW. Beam Current Monitor for the Oak Ridge Electron Linear Accelerator. IEEE Trans Nucl Sci (USA). 1971;18:929. https://doi.org/10.1109/TNS.1971.4326234

20. Shinde RS, Karmarkar MG. High PrecisionCurrent Ferrite Monitors. J de Physique IV (Colloque). 1997;7:159. https:// doi.org/10.1051/jp4:1997157.

21. Blokland W. Beam current monitors, accelerator beam diagnostics. 2009. https://uspas.fnal.gov/materials/09UNM/ BeamCurrentMonitors.pdf.

22. Bogomilov M, Karadzhov Y, Kolev D, Russinov I, Tsenov R, Vankova-Kirilova G, Wang L, Xu FY, Zheng SX, Bertoni R, et al. The MICE Muon Beam on ISIS and the beam-line instrumentation of the Muon lonization Cooling Experiment. J Instrum. 2012;7:P05009.

23. Morenzoni E, Kottmann F, Maden D, Matthias B, Meyberg M, Prokscha Th, Wutzke Th, Zimmermann U. Generation of very slow polarized positive muons. Phys Rev Lett. 1994;72:2793.

24. Alvarez LW, Bradner H, Crawford FS Jr, Crawford JA, Falk-Vairant P, Good ML, Gow JD, Rosenfeld AH, Solmitz F, Stevenson ML, Ticho HK, Tripp RD. Catalysis of Nuclear Reactions by $\mu$ Mesons. Phys Rev. 1957;105:1127.

25. Balin DV, Ganzha VA, Kozlov SM, Maev EM, Petrov GE, Soroka MA, Schapkin GN, Semenchuk GG, Trofimov VA, Vasiliev AA, Vorobyov AA, Voropaev NI, Petitjean C, Gartner B, Lauss B, Marton J, Zmeskal J, Case T, Crowe KM, Kammel P, Hartmann FJ, Faifman MP. High precision study of muon catalyzed fusion in D and HD gas ${ }_{2}$. Phys Part Nuclei. 2011:42:185.

26. Holmlid L. Neutrons from muon-catalyzed fusion and from capture processes in an ultra-dense hydrogen $\mathrm{H}(0)$ generator. Fusion Sci Technol. https://doi.org/10.1080/15361055.2017.1421366.

27. Holmlid L. The solar wind proton ejection mechanism: experiments with ultra-dense hydrogen agree with observed velocity distributions. J Geophys Res Space Phys. 2017;122:7956-62. https://doi.org/10.1002/2017JA024498.

28. Holmlid L. Ultra-dense hydrogen $\mathrm{H}(0)$ as stable dark matter in the Universe: extended red emission spectra agree with rotational transitions in H(0). Astrophys J. 2018;866:107. https://doi.org/10.3847/1538-4357/aadda1.

29. Nordling C, Österman J. Physics handbook. Lund: Studentlitteratur; 1988.

30. Particle Data Group, Tanabashi M, et al. Phys Rev D. 2018;98:030001. 2019 update. URL: http://pdg.lbl.gov.

31. Andersson PU, Lönn B, Holmlid L. Efficient source for the production of ultra-dense deuterium D(-1) for laserinduced fusion (ICF). Rev Sci Instrum. 2011;82:013503.

32. Holmlid L. Laser-induced nuclear processes in ultra-dense hydrogen take place in small non-superfluid $\mathrm{H}_{\mathrm{N}}(0)$ clusters. J Cluster Sci. 2019;30(1):235-42. https://doi.org/10.1007/s10876-018-1480-5.

33. Holmlid L. Energy production by laser-induced annihilation in ultradense hydrogen $\mathrm{H}(0)$. Int J Hydrog Energy. 2021;46:14592-5. https://doi.org/10.1016/j.jijhydene.2021.01.212.

34. Andersson PU, Holmlid L. Fast atoms and negative chain cluster fragments from laser-induced Coulomb explosions in a super-fluid film of ultra-dense deuterium D(-1). Phys Scr. 2012;86:045601. https://doi.org/10.1088/0031-8949/ $86 / 04 / 045601$

35. Meima GR, Menon PG. Catalyst deactivation phenomena in styrene production. Appl Catal A. 2001;212:239.

36. Muhler M, Schlögl R, Ertl G. The nature of the iron oxide-based catalyst for dehydrogenation of ethylbenzene to styrene 2. Surface chemistry of the active phase J Catal. 1992;138:413.

37. Holmlid L. Experimental studies and observations of clusters of Rydberg matter and its extreme forms. J Cluster Sci. 2012;23:5-34. https://doi.org/10.1007/s10876-011-0417-z.

38. Holmlid L, Olafsson S. Laser-induced annihilation: relativistic particles from ultra-dense hydrogen $\mathrm{H}(0)$. High Energy Density Phys. 2021:40:100942. https://doi.org/10.1016/j.hedp.2021.100942.

39. Holmlid L. Leptons from decay of mesons in the laser-induced particle pulse from ultra-dense protium $\mathrm{p}(0)$. Int $J$ Modern Phys E. 2016;25:1650085. https://doi.org/10.1142/S0218301316500853.

\section{Publisher's Note}

Springer Nature remains neutral with regard to jurisdictional claims in published maps and institutional affiliations. 